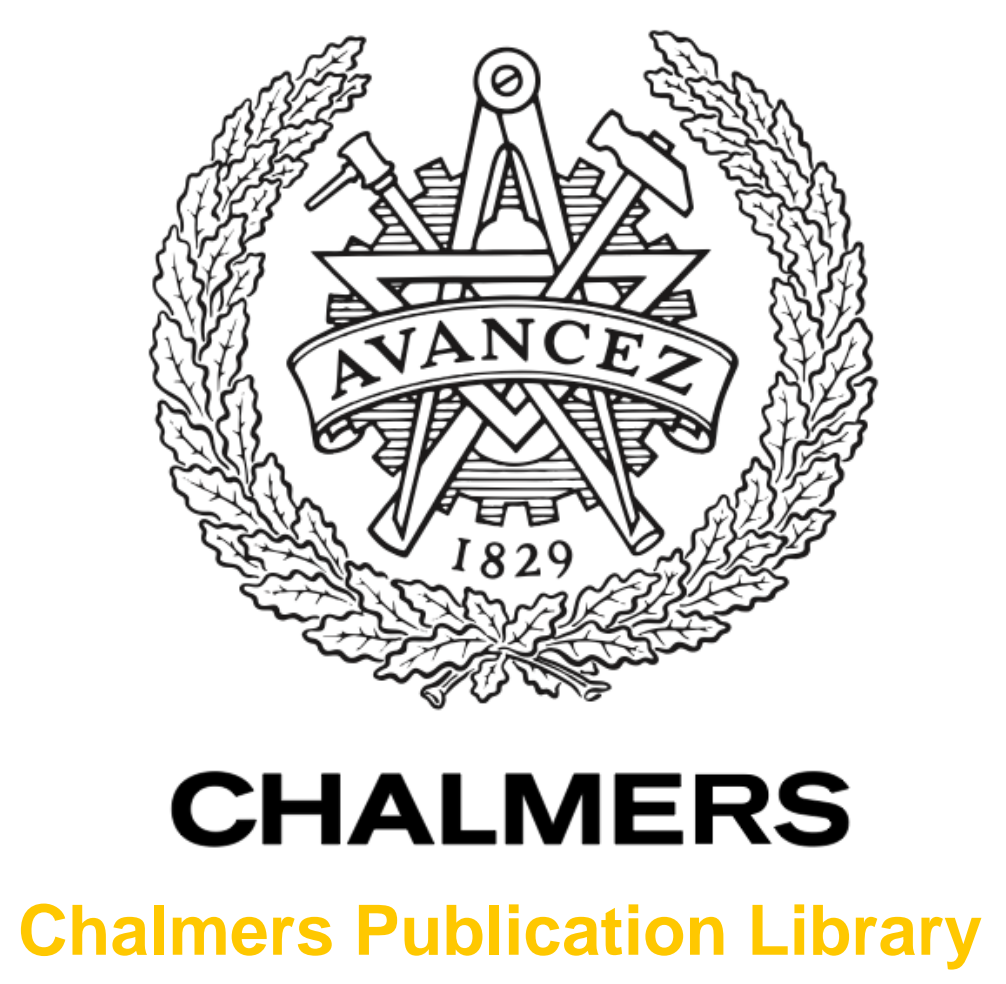

Performance limitations in vehicle platoon control

This document has been downloaded from Chalmers Publication Library (CPL). It is the author's version of a work that was accepted for publication in:

2012 15th International IEEE Conference on Intelligent Transportation Systems, ITSC 2012, Anchorage, AK, 16 - 19 September 2012

Citation for the published paper:

Solyom, S. ; Coelingh, E. (2012) "Performance limitations in vehicle platoon control". 2012 15th International IEEE Conference on Intelligent Transportation Systems, ITSC 2012,

Anchorage, AK, 16 - 19 September 2012 pp. 1-6.

http://dx.doi.org/10.1109/ITSC.2012.6338689

Downloaded from: http://publications.lib.chalmers.se/publication/169852

Notice: Changes introduced as a result of publishing processes such as copy-editing and formatting may not be reflected in this document. For a definitive version of this work, please refer to the published source. Please note that access to the published version might require a subscription.

Chalmers Publication Library (CPL) offers the possibility of retrieving research publications produced at Chalmers University of Technology. It covers all types of publications: articles, dissertations, licentiate theses, masters theses, conference papers, reports etc. Since 2006 it is the official tool for Chalmers official publication statistics. To ensure that Chalmers research results are disseminated as widely as possible, an Open Access Policy has been adopted.

The CPL service is administrated and maintained by Chalmers Library. 


\title{
Performance Limitations in Vehicle Platoon Control
}

\author{
Stefan Solyom and Erik Coelingh
}

\begin{abstract}
One of the major benefits of driving vehicles in controlled, close formations such as platoons is that of reduced air drag. However, this will set hard performance requirements on the system actuators, sensors and controllers of each vehicle. This paper analyzes the effects of fundamental limitations on the longitudinal and lateral control performance of a platoon and the effects on following distance, perceived safety and fuel economy. The trade-off between minimizing fuel consumption and maintaining a safe following distance is analyzed and described. The analysis is based on fundamental properties of linear systems such as Bode's phase area relation. Design guidelines are proposed and results from vehicle testing are presented.
\end{abstract}

\section{INTRODUCTION}

Automated vehicle platoon is a well studied of application in the field of automatic control. Already in the late 80 's, early results on control methods, stability analysis and vehicle testing are presented [11], [5], [14], [10]. The presented platoons are automated such that both the longitudinal direction and the lateral position of the vehicles in the platoons is controlled automatically. One of the most straightforward advantages of such vehicle formation has shown to be the reduction of aerodynamic drag [16]. A direct consequence of this phenomenon is significant reduction of fuel consumption. However, it has been shown that the drag reduction has an exponential dependence on the distance between the vehicles. In order to maximize the fuel economy, inter-vehicle distance needs to be minimized. Inevitably this will increase the performance requirements on the control system. Then an interesting question is at what point is the process (the platoon) to be controlled limiting the achievable performance, i.e. the fundamental limitations on control performance arising from the process to be controlled. The studied scenario is that of steady cruise for the platoon, i.e. steady state following of the vehicle in front. More specifically, the article examines the disturbance rejection properties during cruising in formation.

For such scenarios, the most studied limitations in todays vehicle are those arising from the longitudinal actuation, that is the internal combustion engine and the brake system. These have been recognized in published results for global stability properties of the platoon e.g. string stability analysis [8], [9], [15]. However, to best of the authors knowledge there are no studies that are addressing the influence of fundamental limitation on achievable longitudinal control

This work was partially supported by the European project SARTRE.

The authors are with Volvo Car Corporation, Active Safety and Vehicle Dynamics, SE-40531 Göteborg, Sweden, Tel: +46(0)31595340; ssolyom@volvocars.com, ecoeling@volvocars.com

Erik Coelingh is also with Chalmers University of Technology, Department for Signals and Systems, Göteborg, Sweden. performance locally for each vehicle in the platoon and its effects on the platoon.

Additionally, it will be shown that performance limitations will arise also from the lateral dynamics of the platoon. The considered system configuration is that of forward-looking sensing, a ranging sensor, such as camera placed behind the windshield of the vehicle. This sensor is already available in series production vehicle for collision avoidance functions. It is an economically sound solution to use this sensor also for vehicle platoon control. The disadvantage is however, that in close formation such as platoons, the lane tracking detection is occluded by the preceding vehicle. That is, the lane markings can not be used for lateral control. Since absolute lateral position control through series production GPS is highly inaccurate, the only viable solution is that of following the preceding vehicle. The paper will contribute also in describing the influence of fundamental limitation on lateral control performance for this setup. Moreover, the article will link the limitations on the achievable lateral and longitudinal control performance, i.e. the influence of lateral control performance limitation on the following distance and thus the fuel consumption.

The outline of the paper is as follows, Section 2 is describing the controlled process. Section 3 contains the analytical results of the paper including the design guidelines for formation control as well as some numerical example and design guideline. Section 4 shows the experimental results from vehicle testing.

\section{Vehicle ANd Formation Modeling}

The assumption used in this section is that of linearity of both the controller and the process. This might seem to introduce significant conservativeness in the results, however, the operating ranges the analysis is addressing are rather narrow around an operating point, i.e. steady state cruise, where the linearity assumptions hold. The considered process model to be controlled is shown in Figure 1. It is rather

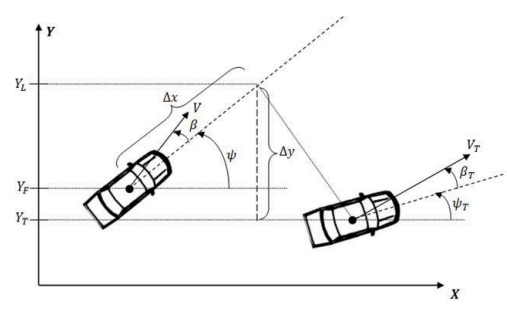

Fig. 1. Host vehicle following a target vehicle. 


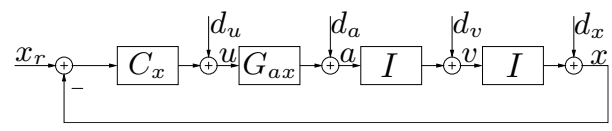

Fig. 2. Vehicle relative longitudinal position driven by a requested longitudinal acceleration. The longitudinal acceleration control loop has a lumped time constant $T_{a x}$. The longitudinal platoon formation controller is $C_{x}(s)$.

simple, and expresses the relative longitudinal and lateral position respectively between two vehicles.

\section{A. The Longitudinal Following}

The transfer function from the desired acceleration to the vehicle position is [7]:

$$
P_{x}(s)=\frac{1}{s^{2}\left(s T_{a x}+1\right)} e^{-s T_{d x}}
$$

where $T_{a x}$ is the time constant of the longitudinal acceleration control. This time constant describes the lumped dynamics of the controlled combustion engine or the controlled braking system, depending on the sign and level of the acceleration. Also communication delays can be considered in the term $T_{d x}$. For later use we define the terms:

$$
G_{a x}(s)=\frac{1}{s T_{a x}+1} e^{-s T_{d x}}, \quad I(s)=\frac{1}{s} .
$$

This can be described with the block diagram in Figure 2 , with the relative position $x$, velocity $v$ and acceleration $a$ states respectively. The corresponding disturbance inputs are $d_{x}, d_{v}, d_{a}$, while the control input $u$ is the requested acceleration of the host vehicle with its respective disturbance $d_{u}$.

\section{B. The Lateral Following}

The model used for describing the lateral following of a target vehicle is based on a linear parameter varying bicycle model [6]. The considered parameter is the longitudinal velocity $v$. However, since the longitudinal velocity is near constant during cruising, the system can be considered to be linear.

$$
\dot{\zeta}=A \zeta+B \delta+w
$$

with

$$
\begin{gathered}
A=\left[\begin{array}{cccc}
-\frac{C_{F}+C_{R}}{m v} & -\frac{l_{F} C_{F}-l_{R} C_{R}}{m v^{2}}-1 & 0 & 0 \\
-\frac{l_{F} C_{F}-l_{R} C_{R}}{J} & -\frac{l_{F}^{2} C_{F}+l_{R}^{2} C_{R}}{J v} & 0 & 0 \\
0 & 1 & 0 & 0 \\
v & L & v & 0
\end{array}\right] \\
B=\left[\begin{array}{llll}
\frac{C_{F}}{m v} & \frac{C_{F} l_{F}}{J} & 0 & 0
\end{array}\right]^{T}
\end{gathered}
$$

and

$$
w=\left[\begin{array}{llll}
0 & 0 & 0 & v\left(\beta_{T}+\Psi_{T}\right)
\end{array}\right]^{T} .
$$

In this case the state vector is formed by: $\zeta=[\beta \dot{\Psi} \Psi \Delta y]^{T}$, i.e. the side slip angle, yaw rate, yaw angle and lateral deviation between the host and target vehicle respectively as depicted in Figure 1. The input signal of the system is the wheel angle $\delta$. The target side slip angle and yaw

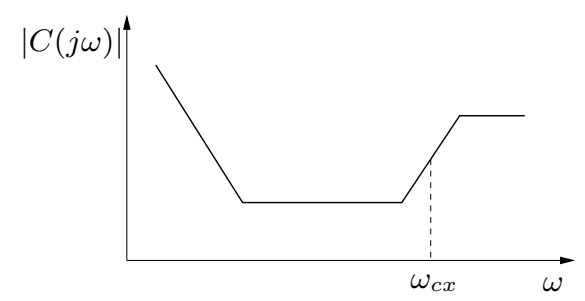

Fig. 3. The gain curve of a longitudinal platoon controller with a crossover frequency $\omega_{c x}$. The maximum high frequency gain is given by $C_{x} \max$ while the minimum gain is denoted $C_{x}$ min.

angle can be considered known in case vehicle to vehicle communication is available, otherwise can be considered as an unknown disturbance. The remaining model parameters are as follows: $C_{F, R}$ front and rear axle cornering stiffness, $l_{F, R}$ distance of COG to front and rear axle, $J$ vehicle moment of inertia, $m$ vehicle mass and $L$ the look-ahead distance. The latter is shown in Figure 1, in the general case by $\Delta x$. It is however undesirable to directly control the wheel angle of the vehicle with the high level target following controller. Therefore an additional control loop for the wheel angle is assumed. The controlled wheel angle is then approximated with a low-pass filter, which now represents the controlled actuator dynamics. Also time delay can be added to the model, that can represent communication delays:

$$
G_{a y}(s)=\frac{1}{s T_{a y}+1} e^{-s T_{d y}}
$$

For later use denote $P_{y}$ the total system transfer function of the following vehicles in (2) and the actuator dynamics. An interesting property of the system is that it can become nonminimum phase as the look-ahead distance is decreased and the vehicle speed is increased [4]. This will play an important role in the shaping of the sensitivity functions.

\section{Limitation of Achievable Control PERFORMANCE}

The tool used for obtaining the analytical results on performance limitation is Bode's phase area formula [3], [1]. The result gives an estimate on the required gain to obtain a certain phase lead. The gain of a controller that achieves good longitudinal control performance is shown in Figure 3. Since the lateral and longitudinal processes are similar, the same controller gain requirements would apply also for the lateral control. Therefore, during the deduction of the general analytical results, for ease of presentation consider a generic controller $C$, the results hold for both lateral and longitudinal case. Using the phase area formula, it can be shown [1], that the maximum high frequency gain of the controller is:

$$
C_{\max }^{*}=e^{2 \gamma \arg C\left(j \omega_{c}\right)}
$$

where $\gamma$ is a design parameter with a reasonable value of 1 . After scaling the controller such that the desired crossover frequency, $\omega_{c}$, is achieved the maximum high frequency gain 
is:

$$
C_{\text {max }}=\frac{\sqrt{e^{2 \gamma \arg C\left(j \omega_{c}\right)}}}{\left|P\left(j \omega_{c}\right)\right|}=\frac{e^{\gamma \arg C\left(j \omega_{c}\right)}}{\left|P\left(j \omega_{c}\right)\right|}
$$

Similarly the minimum controller gain can be computed to be:

$$
C_{\text {min }}=\frac{1}{\left|P\left(j \omega_{c}\right)\right| e^{\gamma \arg C\left(j \omega_{c}\right)}}
$$

\section{A. The Longitudinal Control}

For a given phase margin $\phi_{m}$ and the considered process, the maximum high frequency gain of the controller becomes:

$$
\begin{aligned}
& C_{x \max }=\omega_{c x}^{2} \sqrt{\omega_{c x}^{2} T_{a x}^{2}+1} e^{\gamma\left(\phi_{m}+\operatorname{atan} \omega_{c x} T_{a x}+\omega_{c x} T_{d x}\right)} \\
& C_{x \min }=\omega_{c x}^{2} \sqrt{\omega_{c x}^{2} T_{a x}^{2}+1} \frac{1}{e^{\gamma\left(\phi_{m}+\operatorname{atan} \omega_{c x} T_{a x}+\omega_{c x} T_{d x}\right)}}
\end{aligned}
$$

In the following a set of sensitivity functions are defined that are relating different disturbances to specific input and output signals.

1) The Longitudinal Position Input Sensitivity Function: The position input sensitivity function is given by:

$$
S_{x u}(s)=\frac{U(s)}{D_{x}(s)}=\frac{C_{x}(s)}{1+P_{x}(s) C_{x}(s)}
$$

It is clear that for high frequencies, typical for measurement noise the high frequency maximum gain for $S_{x u}$ is given by (6). This sensitivity function describes the influence of position uncertainties on the control signal and in turns the fuel economy.

2) The Longitudinal Acceleration Input Sensitivity Function: The acceleration input sensitivity function is defined as:

$$
S_{a u}(s)=\frac{U(s)}{D_{A}(s)}=\frac{I^{2}(s) C_{x}(s)}{1+P_{x}(s) C_{x}(s)}=I(s)^{2} \frac{T_{x}(s)}{P_{x}(s)}
$$

Using the same argument as above, around the crossover frequency can be written:

$$
\max \left|S_{a u}\right|<M_{T} \sqrt{\omega_{c x}^{2} T_{a x}^{2}+1}
$$

This sensitivity function gives a measure of the influence of acceleration noise and error to the controller output and thus the fuel consumption and comfort.

3) The Longitudinal Load Sensitivity Function: The load sensitivity function is given by:

$$
S_{u x}(s)=\frac{X(s)}{D_{U}(s)}=\frac{P_{x}(s)}{1+P_{x}(s) C_{x}(s)}=\frac{T_{x}(s)}{C_{x}(s)}
$$

and describes the output sensitivity for a disturbance in the control signal. For controllers with substantial phase lead the maximum of $S_{u x}$ occurs at frequencies below the crossover frequency $\omega_{c x}$. In this frequency range, for a good design, $\left|T_{x}\right| \approx 1$, and assuming the controller gain curve as shown in Figure 3, the maximum gain is:

$$
\max \left|S_{u x}\right| \approx \frac{1}{C_{x \min }}=\left|P_{x}\left(j \omega_{c x}\right)\right| e^{\gamma \arg C_{x}\left(j \omega_{c x}\right)}
$$

For the plant in (1) this means:

$$
\max \left|S_{u x}\right| \approx \frac{1}{\omega_{c x}^{2} \sqrt{\omega_{c x}^{2} T_{a x}^{2}+1}} e^{\gamma\left(\phi_{m}+\operatorname{atan} \omega_{c x} T_{a x}+\omega_{c x} T_{d x}\right)}
$$

The sensitivity functions for the studied application would describe the changes in the platoon gaps with respect to a disturbance introduced on the acceleration request. This in practice would mean a disturbance on the acceleration control loop, e.g. time varying uncertainties on the road load estimation.

4) The Longitudinal Acceleration Output Sensitivity Function: The acceleration output sensitivity function is defined as:

$$
S_{a x}(s)=\frac{X(s)}{D_{A}(s)}=\frac{I(s)^{2}}{1+P_{x}(s) C_{x}(s)}=\frac{S_{u x}(s)}{G_{a x}(s)}
$$

Using the same argument as above, at $\omega_{c x}$ the relation becomes:

$$
\begin{gathered}
\max \left|S_{a x}\right| \leq \frac{1}{\left|G_{a x}\left(j \omega_{c x}\right)\right|} \max \left|S_{u x}\right| \\
\approx \frac{1}{\omega_{c x}^{2}} e^{\gamma\left(\phi_{m}+\operatorname{atan} \omega_{c x} T_{a x}+\omega_{c x} T_{d x}\right)} .
\end{gathered}
$$

For the studied application, this sensitivity function describes effects of acceleration disturbances on the controlled intervehicle gap. It is therefore a very useful tool for analyzing the following distance in the platoon.

Similarly to the above presented sensitivity functions, the velocity sensitivity functions can be defined [13].

\section{B. The Lateral Control}

The following lateral sensitivity functions are defined:

1) The Lateral Input Sensitivity Function:

$$
S_{y u}(s)=\frac{U(s)}{D_{y}(s)}=\frac{C_{y}(s)}{1+P_{y}(s) C_{y}(s)}
$$

where $C_{y}$ is the lateral position following controller. Just as in the longitudinal case, the maximum gain of the sensitivity function is given by (4). The interpretation of $S_{y u}$ is that of the maximum wheel angle (and in turns steering wheel angle) that will be actuated for a given lateral deviation. This has a practical importance for the comfort and perceived safety of the passengers in case of disturbances on the lateral position measurement. Also the corresponding output sensitivity function has an important interpretation.

2) The Lateral Load Sensitivity Function: The load sensitivity function is given by:

$$
S_{u y}(s)=\frac{\Delta Y(s)}{D_{U}(s)}=\frac{P_{y}(s)}{1+P_{y}(s) C_{y}(s)}
$$

with

$$
\max \left|S_{u y}\right| \approx \frac{1}{C_{y \min }}=\left|P_{y}\left(j \omega_{c y}\right)\right| e^{\gamma \arg C_{y}\left(j \omega_{c y}\right)}
$$

where $\omega_{c y}$ is the chosen lateral crossover frequency. The practical relevance of this sensitivity function is that of the maximum lateral deviation in case of disturbances on the wheel angle, as it would be in case of uneven roads, torque steer. 


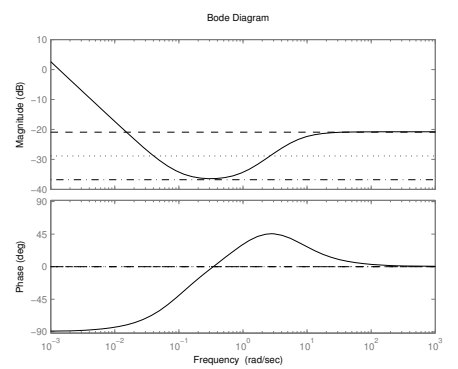

Fig. 4. Controller phase-gain plot and the bounds $C_{y \max }$ and $C_{y \min }$.

3) The Lateral Acceleration Output Sensitivity Function: The acceleration output sensitivity function is defined as:

$$
S_{a y}(s)=\frac{\Delta Y(s)}{D_{A}(s)}=\frac{P_{y a}(s)}{1+P_{y}(s) C_{y}(s)}
$$

where $P_{y a}(s)$ is the open loop process transfer function from $D_{A}(s)$ to $\Delta Y(s)$. It can be obtained using the input matrix:

$$
B_{a}=\left[\begin{array}{llll}
1 / v & 0 & 0 & 0
\end{array}\right]^{T} \text {. }
$$

Define the transfer function:

$$
H(s)=\frac{Z P_{y a}(s)}{Z P_{y}(s)}
$$

formed by the zeros of $P_{y}(s)$ and $P_{y a}(s)$ respectively. Since the maximum gain of the considered sensitivity function is obtained around the crossover frequency, one can write:

$$
\max \left|S_{a y}\right| \leq\left|H\left(j \omega_{c y}\right)\right| \max \left|S_{u y}\right| \approx \frac{\left|H\left(j \omega_{c y}\right)\right|}{C_{y \min }}
$$

This sensitivity function quantifies the effect of lateral acceleration disturbances such as side wind, road banking.

\section{Numerical Example}

To get a feel of the above derived bounds consider the following lateral position controller, i.e. the controller input signal is the lateral deviation $\Delta y$ :

$$
C_{y}(s)=0.0135\left(\frac{0.85 s}{0.15 s+1}+\frac{0.1}{s}+1.1\right) .
$$

For a longitudinal controller refer to [13]. For $T_{a y}=0.1$ and $T_{d x}=0$ it will guarantee a phase margin of $43.7^{\circ}$ with a crossover frequency of $2.65 \mathrm{rad} / \mathrm{s}$. The considered vehicle speed is $25 \mathrm{~m} / \mathrm{s}$.

Figure 4 shows the phase-gain plot of the chosen controller $C_{y}$, with the respective maximum high frequency and minimum gain bounds. To limit the length of the article, only the output sensitivity functions are considered, nevertheless similar results are obtained for the input sensitivity functions. Figure 5 depicts the considered output sensitivity functions with the computed bounds and approximations. It can be seen that the bounds and approximated values are showing rather good accuracies, despite of a rather simple controller. These bounds will be better as the controller complexity is increased and an ideal loop-shape is achieved. Figure 6

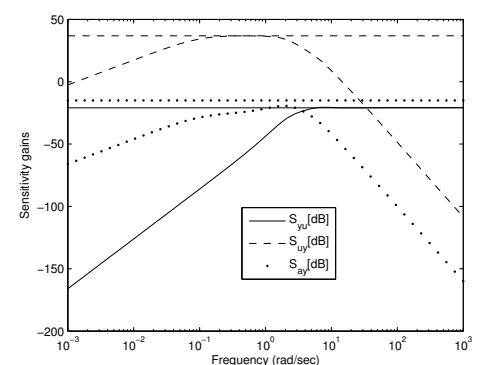

Fig. 5. Output Lateral Sensitivity Functions and their respective bounds.

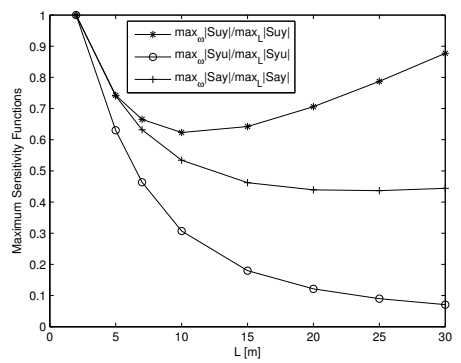

Fig. 6. The approximated values of the normalized Maximum Output Sensitivity Functions with respect to the look-ahead distance $L$. The normalization factor is the highest value of each maximum sensitivity function computed in the given look-ahead distance interval.

show the values of the normalized estimated bounds with respect to the look-ahead distance $L$. The normalization factor is the highest value of each maximum sensitivity function computed in the given look-ahead distance interval. The considered parameters in computing the bounds are $\omega_{c y}=2.5$ and $\phi_{m}=45^{\circ}$. The plot gives a quantitative measure on the following control performance degradation as the following distance is reduced.

\section{Consequences of Performance Limitations}

The sensitivity functions derived in the previous sections can be used to define how disturbances in the controlled system affect the motion of the following vehicle as well as the fuel economy.

In order to address the safety aspects of automated vehicle following, two proximity margins of a platoon vehicle are defined. The longitudinal proximity margin provides a relation between the desired inter-vehicle distance $x_{r}$ and the position error $\Delta x$ :

$$
\mathcal{P}_{m x}=1-\frac{\min \left(|\Delta x|, x_{r}\right)}{x_{r}},
$$

where $\Delta x=x-x_{r}$. When the position error $\Delta x$ is much smaller than the desired inter-vehicle distance there is a large longitudinal proximity margin, i.e. a low risk that vehicles will collide.

Similarly the lateral proximity margin provides a relation between the actual lateral deviation $\Delta y$ and the maximum 


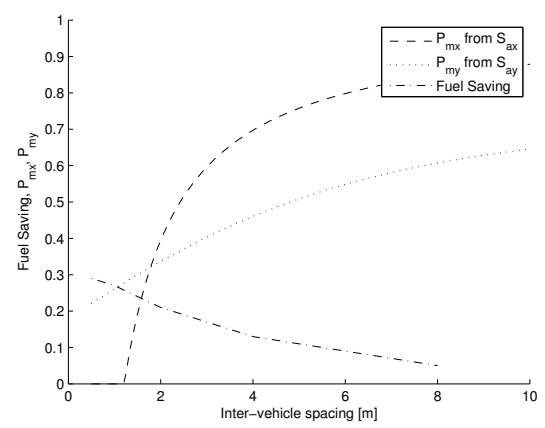

Fig. 7. Trade-off between fuel economy and proximity margin.

allowed lateral deviation $\Delta y_{\max }$.

$$
\mathcal{P}_{m y}=1-\frac{|\Delta y|}{\Delta y_{\max }},
$$

Here, $\Delta y_{\max }$ is not a tuning parameter as in the longitudinal case, but rather a parameter that is determined by the lane or wake width. The latter is important in order to maintain the slip stream effect in the platoon. A proper choice for $\Delta y_{\max }$ would be between $[0.25,0.5] \mathrm{m}$.

Given the maximum disturbances and the maximum of the output sensitivity functions one can compute the maximum actual achievable longitudinal and lateral position errors, such as:

$$
\begin{aligned}
\|\Delta x\|_{\infty} & =\max \left(\max \left|S_{u x}\right|\left\|d_{u}\right\|_{\infty}, \max \left|S_{a x}\right|\left\|d_{a}\right\|_{\infty}\right) \\
\|\Delta y\|_{\infty} & =\max \left(\max \left|S_{u y}\right|\left\|d_{u}\right\|_{\infty}, \max \left|S_{a y}\right|\left\|d_{a}\right\|_{\infty}\right)
\end{aligned}
$$

This way a worst case proximity margin can be computed. Using the same principle on the maximum disturbances, the input sensitivity functions can be plotted as well. These functions indicate the maximum effect of disturbances on the control signals. Figure 7 shows the proximity margins $\mathcal{P}_{m x}$ and $\mathcal{P}_{m y}$ rising from acceleration disturbances whereby the actuator time-constants are selected as $T_{a x}=0.1, T_{a y}=$ 0.1 and $\left\|d_{a x}\right\|_{\infty}=0.5,\left\|d_{a y}\right\|_{\infty}=0.5$. The dash-doted line shows the fuel economy characteristics with respect to inter-vehicle spacing reported in [11]. Although the fueleconomy curve is probably not matched in $\omega_{c x}$ and $T_{a x}$ of the longitudinal sensitivity function curves, it can be seen that there is a trade-off between fuel consumption and proximity margins when selecting a desired inter-vehicle gap. In particular, for the given $\omega_{c x}, \omega_{c y}$ and disturbances, a longitudinal proximity margin of around 0.8 and a lateral proximity margin of 0.5 can be obtained on the longitudinal and lateral acceleration output sensitivity function for a intervehicle spacing of 6 meters, with a $T_{a x}=0.1$ and $T_{a y}=0.1$ seconds.

The relations above can be used both for analysis and design of a platoon system taking into account several different type of disturbances, e.g. acceleration, position, velocity, etc. In case the vehicle is given and the maximum disturbances and the actuator time constants are known, the relations above can be used to design the platoon controller, i.e. determine the cross-over frequencies $\omega_{c x}$ and $\omega_{c y}$ :

Design Procedure 1: Existing vehicle

- For a given vehicle where the actuator time constants and the maximum disturbances are known, as well as the fuel consumption as a function of the inter-vehicle spacing.

- Select a the desired inter-vehicle gap $y_{r}$ and the desired maximum longitudinal control signal $\|u\|_{\infty}$. Assume phase margins $\phi_{m x}$ and $\phi_{m y}$ as well as desired minimum proximity margins $\mathcal{P}_{m x}$ and $\mathcal{P}_{m y}$.

- Calculate the allowable maxima of the sensitivity functions.

- Calculate the limits on $\omega_{c x}$ and $\omega_{c y}$.

Alternatively one calculates the required vehicle dynamics for a desired platoon performance:

\section{Design Procedure 2: Desired performance}

- For a given platoon performance the desired intervehicle gap $y_{r}$ and the desired maximum longitudinal control signal $\|u\|_{\infty}$ are known.

- Assume maximum longitudinal and lateral disturbances $\left\|d_{i}\right\|_{\infty}$. Assume phase margins $\phi_{m x}$ and $\phi_{m y}$, as well as desired minimum proximity margins $\mathcal{P}_{m x}$ and $\mathcal{P}_{m y}$.

- Calculate the maxima of all sensitivity functions as a function of $T_{a x}, \omega_{c x}, T_{a y}$ and $\omega_{c y}$.

- Calculate the limits on the cross-over frequencies $\omega_{c x}$ and $\omega_{c y}$, and the time constants $T_{a x}, T_{a y}$ as well.

\section{EXPERIMENTAL RESUlTS}

\section{A. Experimental Setup}

The vehicle used for testing is a prototype Volvo S60 vehicle with a 3.2 liters turbocharged engine. The vehicle platoon control algorithms is physically implemented on the Forward Sensing Module (FSM). However, except the most part of the sensor processing, the formation control algorithm is run from a dSPACE development environment, with a dSPACE real-time computer. The vehicle acceleration control is located on an additional control unit, the engine control module (ECM). Thus the control system is distributed between different control modules. These modules communicate through a CAN communication buss. This communication delay can be included in $T_{d x}$ and $T_{d y}$.

\section{B. Test Results}

Next, the tests of a longitudinally controlled vehicle platoon are presented. The lateral controller tests where not yet available at the moment this report was written. The controller used is a autonomous range and range rate based controller, i.e. it relies on data exclusively from on-board sensors.

The original aim of the tests is to examine the effect of actuator lag on the control performance of the formation. However due to architectural reasons it is not possible to change the dynamics of the longitudinal acceleration control of the test vehicle, $T_{a x}$. Therefore, the bandwidth of the formation control $\omega_{c x}$ is increased. Three controller parameter sets were tested in the vehicle, tuned to three different 

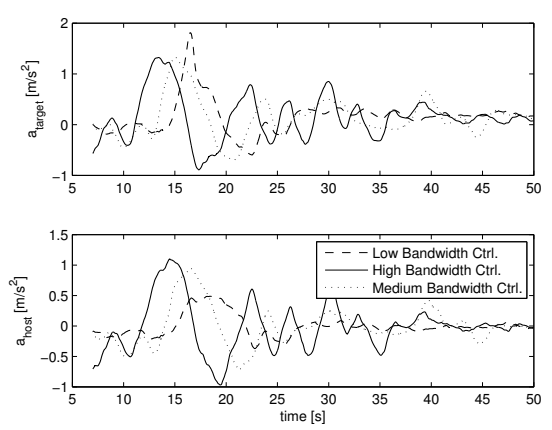

Fig. 8. Target and host acceleration during the test sequence.

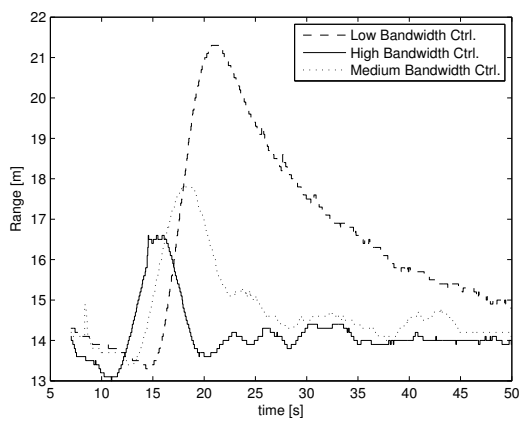

Fig. 9. Ranges for the three controller parameter sets. The solid line shows the controller with the highest bandwidth.

bandwidths. The highest bandwidth controller is shown with solid lines.

Figure 8 shows the acceleration profile during the test sequences, both for the target vehicle, i.e. the disturbance and the host vehicle. The target vehicle is manually driven and the disturbance acceleration is generated by engine braking. During the testing the target vehicle aimed to generate the same disturbance acceleration during each test sequences.

As expected the response of the highest-bandwidth controller has a highest amplitude, i.e. highest acceleration request. This will have an immediate effect on the range as shown in Figure 9, having the smallest range deviation from the set-point than the other controllers. Figure 10 shows the instantaneous normalized fuel consumption during the test sequence. The highest bandwidth controller has the highest fuel usage. This is most obvious during the acceleration phase between 12 and 20 seconds.

\section{CONCLUSions}

Fundamental limitations for longitudinal and lateral vehicle platoon control have been identified and studied. The scenarios considered are those of steady state cruising around a vehicle speed set-point while the system is exposed to small signal disturbances. It is quantified how the actuator lags, time delays and the actual following distance will limit the disturbance rejection performance of the system. The paper describes the trade-off between minimizing fuel consumption and maintaining a safe following distance. It also gives a

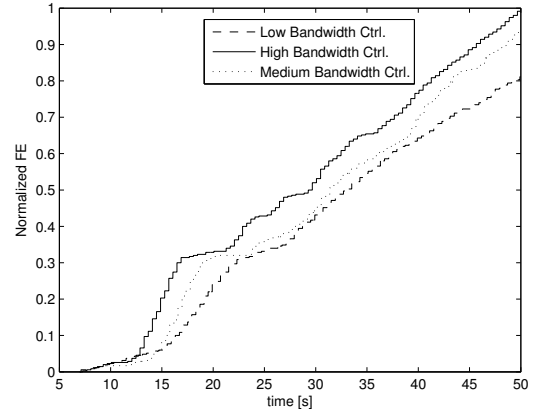

Fig. 10. Normalized fuel economy counters for the three tunes. The highest bandwidth controller has the highest fuel usage.

design procedure for the longitudinal and lateral control for a desired following distance. All the quantitative results derived in the paper are independent of any particular controller structure, as they are based solely on the fundamental limitations arising from the controlled plant.

\section{REFERENCES}

[1] Åström, K. J.: "Limitations on control system performance." In European Control Conference. 1997, Brussels, Belgium.

[2] Åström, K. J., H. Panagopoulos, T. Hägglund: "Design of PI controllers based on non-convex optimization." Automatica, 1998, 34:5, pp. 585-601.

[3] Bode, H.W.: "Network Analysis and Feedback Amplifier Design" Van Nostrand, New York, 1945.

[4] Idelchi, A., Salamah, B. B.: "Lateral Control of Vehicle Platoons", Master Thesis report, Chalmers University, Gothenburg, Sweden, 2010.

[5] Ioannou, P.A., Chien, C.C. :"Autonomous Intelligent Cruise Control", In IEEE Transactions on Vehicular Technology, 1993, pp. 657-672.

[6] Pacejka, H.B. (2006): Tyre and Vehicle Dynamics, Second Edition, Elsevier 2006.

[7] Rajamani, R.: "Vehicle Dynamics and Control", Springer, Mechanical Engineering Series, 2005.

[8] Rajamani, R., Shladover, S.E.: "An experimental comparative study of autonomous and co-operative vehicle follower control system", In Transportation Research Part C9, 2001, pp. 15-31.

[9] Rajamani, R., Zhu, C.: "Semi-Autonomous Adaptive Cruise Control Systems", In IEEE Transactions on Vehicular Technology, 2002, pp. 1186-1192.

[10] Shaw, E., Hedrick, J.K. : "Controller Design for String Stable Heterogeneous Vehicle Strings", In Proceedings of the 46th IEEE Conference on Decision and Control, 2007, pp. 2869-2875.

[11] Shladover, S. et.al : "Automatic Vehicle Control Developments in the PATH Program", In IEEE Transaction on Vehicular Technology, 1991, pp. 114-130.

[12] Solyom, S., Ingimundarson, A. (2002): "A synthesis method for robust PID controllers for a class of uncertain systems." Asian Journal of Control, 4:4, pp. 381-387.

[13] Solyom, S., Coelingh, E. and Carlsson, B.: "Performance Limitations in Longitudinal Control of Vehicle Platoons", In Proceedings of the $11^{\text {th }}$ Advanced Vehicle Control Conference 2012.

[14] Swaroop, D. et.al.: "A Comparison of Spacing and Headway Control Laws for Automatically Controlled Vehicles", In Vehicle System Dynamics, 1994, pp.597-625.

[15] Swaroop, D., Hedrick, J.K.: "String Stability of Interconnected Systems", In IEEE Transactions on Automatic Control, 1996, pp.349357.

[16] Zabat, M. et.al: "The Aerodynamic Performance of Platoons: Final Report", California PATH Research Report, UCB-ITS-PRR-95-35, 1995. 